\title{
Evidence That the Neural Pathways Involved in Backward Conditioning Are Different from Those Involved in Forward Conditioning
}

\author{
Stephen M. Onifer a and Russell G. Durkovic \\ Department of Physiology, SUNY Health Science Center at Syracuse, Syracuse, New York 13210
}

\begin{abstract}
Effects of forward and backward conditioned-unconditioned stimulus (CS-US) intervals on classical conditioning of the flexion reflex were examined in a spinal cat preparation. A less intense conditioned stimulus (CS) was employed (activation of A-alpha cutaneous fibers) compared to that of a previous study (activation of both A-alpha and A-delta cutaneous fibers). Interstimulus intervals (ISIs) ranging from +3.0 to $-3.0 \mathrm{sec}$ were examined in 9 experimental groups, and results contrasted to those of an explicitly unpaired control group. The ISI of $-0.25 \mathrm{sec}$ produced optimal backward excitatory conditioning, paralleling the previous results. However, in contrast to the previous study, no conditioning was observed in any of the forward ISI groups. These observations support the hypothesis that backward and forward conditioning may be fundamentally different phenomena, induced by CS activation of different spinal reflex pathways: backward conditioning involves spinal reflex pathways activated by A-alpha cutaneous fibers of the CS, while forward conditioning involves spinal reflex pathways activated by A-delta cutaneous fibers of the CS.
\end{abstract}

In a recent study it was observed that the interval between the onset of trains of electrical stimuli delivered to 2 different cutaneous nerves was a determining factor in the development of classically conditioned flexion reflex facilitation in the spinal cat (Durkovic and Damianopoulos, 1986). Thus, excitatory conditioning was observed for certain forward interstimulus intervals (ISIs) [conditioned stimulus (CS) onset preceded unconditioned stimulus (US) onset] and certain backward ISIs (US onset preceded CS onset), but not for others. In that study, CS and US intensities were adjusted to evoke maximal activation of A-alpha (large myelinated) and A-delta (small myelinated) cutaneous afferent fibers. While maximal levels of conditioned reflex facilitation were similar for forward conditioning (FC) and backward conditioning (BC), extinction of the facilitation occurred more readily for $\mathrm{BC}$ than for $\mathrm{FC}$. This behavioral difference suggested that $\mathrm{BC}$ and $\mathrm{FC}$ might be the result of different neural mechanisms.

\footnotetext{
Received Sept. 26, 1986; revised June 8, 1987; accepted Aug. 10, 1987.

The authors gratefully acknowledge the expert technical assistance of Clancy Leahy and Brian Young, and the manuscript preparation by Terri McClelland. Dr. Ernest Damianopoulos made helpful comments on early versions of the manuscript. Supported by NSF grants BNS 80-23943 and BNS 84-15917.

Correspondence should be addressed to Dr. R. G. Durkovic at the above address.

a Present address: Department of Physiology and Biophysics, Indiana University of School of Medicine, Indianapolis, IN 46223.

Copyright (C) 1988 Society for Neuroscience $0270-6474 / 88 / 020502-06 \$ 02.00 / 0$
}

The present experiments were conducted as a further test of this hypothesis, using a lower-intensity $\mathrm{CS}$ that resulted in activation of only A-alpha cutaneous fibers, rather than both A-alpha and A-delta fibers. The results support the possibility that $B C$ involves activation of different spinal reflex pathways from those involved in $\mathrm{FC}$, and, together with the behavioral differences between $\mathrm{BC}$ and $\mathrm{FC}$ noted earlier, suggest that forward and backward excitatory conditioning may be products of different neural processes. These findings have important implications for theories of associative learning, and also provide insights concerning the potential neural mechanisms that may be responsible for the form of the ISI function curve.

Short notes dealing with preliminary results of this study have appeared previously (Durkovic and Onifer, 1985; Durkovic, 1986).

\section{Materials and Methods}

Eighty adult male and female cats were used to form onc control and 9 experimental treatment groups ( 8 animals/group). The animals were anesthetized with ether and, following tracheal cannulation, were provided a mixture of $1-2 \%$ halothane in a $2: 1$ mixture of nitrous oxide and oxygen using a Harvard constant-volume ventilator. Cannulation of the right carotid artery (to monitor blood pressure) and ligation of the left carotid artery followed. A laminectomy was performed and the spinal cord sectioned at T-10 with a thermal cautery. At this point, the halothane was discontinued and after $1 \mathrm{~min}$ the animals were anemically decerebrated by clamping the vertebral arteries. The nitrous oxideoxygen mixture was then discontinued and the animals were artificially respirated with room air. An end-tidal $\mathrm{CO}_{2}$ of $2-4 \%$ and arterial blood pressure above $60 \mathrm{mmHg}$ were considered acceptable levels. Up to 10 $\mathrm{ml} / \mathrm{kg}$ of plasma expander (Rheomacrodex; $10 \%$ in normal saline) was administered to help maintain blood pressure. Rectal temperature of $37^{\circ} \mathrm{C}$ was maintained by a heating pad, and Ringer's lactated solution at $10 \mathrm{ml} / \mathrm{hr}$ was administered through a forelimb vein throughout all subsequent procedures.

The animals' hindquarters were fixed in place with a clamp on the sacral vertebral processes and the left hindlimb was rigidly fixed with a femur pin and foot clamp. The cutaneous superficial peroneal and saphenous nerves were isolated from surrounding tissue and placed over hook-shaped bipolar stimulating and recording electrodes. A mineral oil bath formed from surrounding loose skin housed the exposed nerves and electrodes. The distal tendon of the left tibialis anterior (TA) muscle was severed and attached to a transducer, which converted mechanical tension to recording-pen deflection. Resting tension was 50-80 gm.

Following surgery, each of the 80 animals was allocated randomly to one of the 10 groups. Results from 6 additional animals had to be discarded because the physiological criteria for acceptability were not met. Of these, 4 animals did not meet the initial response criteria for reflex activity (see below). For 2 animals the afferent nerve recordings were not stable over training (see below).

The CS was a $1.5 \mathrm{sec}$ train of $0.2 \mathrm{msec}$ electrical pulses at $10 / \mathrm{sec}$ applied to the exposed saphenous nerve. The US was a $0.5 \mathrm{sec}$ pulse train of $0.2 \mathrm{msec}$ pulses at $30 / \mathrm{sec}$ applied to the exposed superficial 


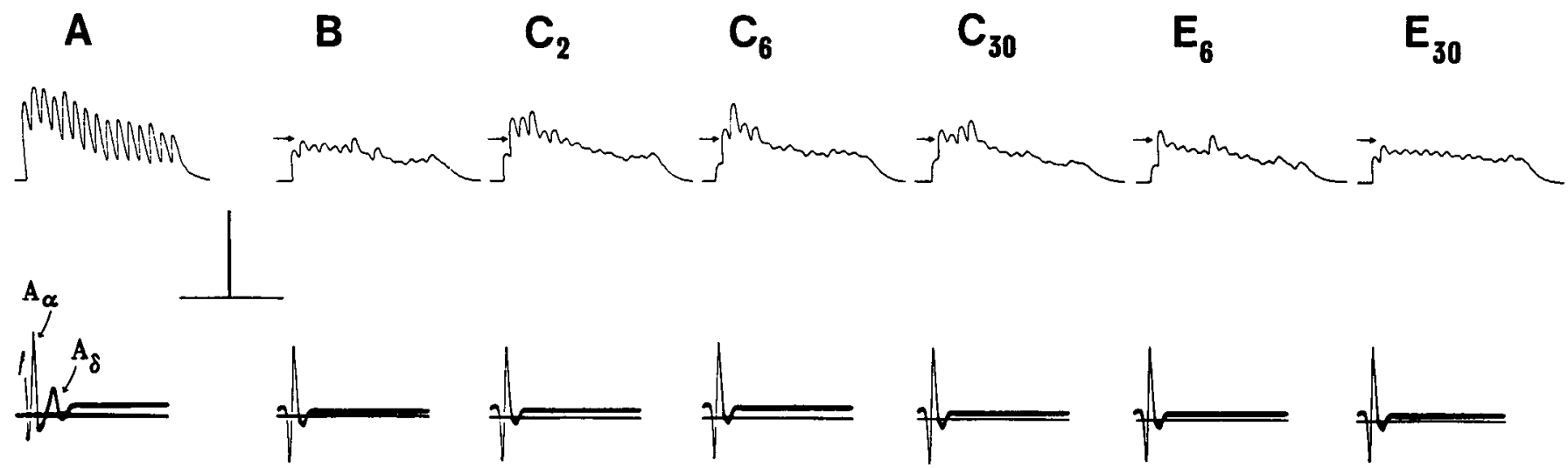

Figure 1. Responses to saphenous nerve stimulation from a representative conditioning animal (ISI, -0.25 sec). Selected TA muscle tension recordings (upper traces) and corresponding superimposed saphenous nerve recordings (lower traces). Other (horizontal) trace with nerve recording is the channel used for superficial peroneal nerve recordings. $A$, Test response to $10 \mathrm{~Hz}$ electrical stimulation at an intensity supramaximal for A-alpha and A-delta fiber activation. $B-E$, Responses to conditioned stimuli (stimulus intensity just below threshold for A-delta fiber activation). Arrows represent the baseline control TA response level. $B$, Baseline control response. $C$, Probe CS-alone responses following paired conditioning trials $\mathrm{C}_{2}, \mathrm{C}_{6}$, and $\mathrm{C}_{30} . E$, Extinction trials $\mathrm{E}_{6}$ and $\mathrm{E}_{30}$. Calibration: Upper traces $-400 \mathrm{gm}, 1 \mathrm{sec}(A) ; 200 \mathrm{gm}, 1 \mathrm{sec}(B-E)$. Lower traces $-1.05 \mathrm{mV}$, $3.33 \mathrm{msec}(A) ; 0.525 \mathrm{mV}, 3.33 \mathrm{msec}(B-E)$.

peroneal nerve. The intensity of the CS was set just below threshold for activation of A-delta cutaneous fibers (Hunt and McIntyre, 1960). Such a CS activated between 55 and $95 \%$ of the total A-alpha (also known as A-beta or A-alpha beta) fibers of the saphenous nerve, as estimated from the height of the A-alpha compound nerve action potential (compared to that evoked by supramaximal A-alpha and A-delta nerve activation). The intensity of the US was set to activate A-alpha and A-delta cutaneous afferents supramaximally, but was below $\mathrm{C}$ fiber threshold. By monitoring the compound nerve action potentials from the saphenous and superficial peroneal cutaneous nerves, constant afferent nerve inputs were ensured over the duration of each experiment (e.g., Durkovic, 1975, fig. 2; Misulis and Durkovic, 1984, fig. 1).

Treatment procedures were initiated after a minimum $2 \mathrm{hr}$ recovery and stabilization period following anemic decerebration. A test CS, stimulation of the saphenous nerve at A-alpha and A-delta (supramaximal) intensity $(10 / \mathrm{sec}$ for $1.5 \mathrm{sec})$ and a test US were administered separately at $1 \mathrm{~min}$ intervals to make sure that stimulating and recording instruments were functioning at appropriate intensity and gain levels, respectively. Any subject failing to respond to the (A-alpha and A-delta) saphenous nerve stimulus train with a reflex of at least $100 \mathrm{gm}$ in amplitude, and to the test US with a reflex of at lcast $350 \mathrm{gm}$ in amplitude, was considered unacceptable because of the possibility of nerve, muscle, or spinal cord dysfunction as a consequence of the surgical procedures (cf. Patterson, 1975, p. 87). Five minutes later, 5 CS-alone trials were administered at $1 \mathrm{~min}$ intervals to establish the $100 \%$ baseline reference level of responding. One minute later, CS presentations in acquisition were presented at $1 \mathrm{~min}$ constant intertrial intervals (ITIs) for all animals.

Subjects in each group received 30 CS-US presentations during acquisition. The CS-US interval varied depending on group assignment but was constant for all subjects within a group. The intervals were (CS onset to US onset): $0.25,0.5,1.0$, and $3.0 \mathrm{sec}$ for the forward conditioning groups; $-0.25,-0.5,-1.0$, and $-3.0 \mathrm{sec}$ for the backward conditioning groups; and $0.0 \mathrm{sec}$ for the simultaneous conditioning group. Control treatment consisted of unpaired CS-US presentations with a $30 \mathrm{sec}$ CS-US interval. Several other types of unpaired (sensitization) control paradigms have been shown to lead to results similar to those induced using this procedure (e.g., Misulis and Durkovic, 1984, p. 89). Following acquisition, all animals received 30 additional CS-alone test trials at $1 \mathrm{~min}$ intervals to test for extinction.

For all treatments the response measured was the maximum tension developed by the TA muscle during the first second of the response to the CS. In order to monitor conditioned responding during acquisition, all animals were given CS-alone "probe" trials 1 min after the CS of CS-US trials 2, 6,12,18,24, and 30. Probe trials were needed because the measure of the animal's response to the CS alone could not be obtained in those groups in which the CS and US were presented closely together, owing to the response to the US. These 6 probe responses were averaged and expressed as percentages of the initial 5 trial CS-alone pretreatment baseline responses. The means of the $30 \mathrm{CS}$-alone extinction responses following acquisition were expressed in the same manner. The reflex responses to pretreatment CS-alone trials (mean of all animals, $209 \mathrm{gm})$ did not vary among groups $(F(9,63)=1.023, p>0.25)$. The percentage changes from pretreatment baseline were analyzed by Dunnett's $t$ test, which is designed for tests of multiple experimental group differences from a single control group (Edwards, 1968). A supplementary analysis of variance (Lundquist, 1953) was also conducted to obtain reliability estimates of simple, interactive, and specific differences among certain forward, backward, and control treatment groups.

\section{Results}

Backward conditioning treatment produced flexion reflex facilitation, with optimum facilitation in the $-0.25 \mathrm{sec}$ ISI group. Representative muscle tension and nerve recordings are shown in Figure 1. The overall mean group responses for acquisition are presented in Figure 2. Dunnett's $t$ test revealed a significant difference $(p<0.05)$ compared to control group response levels for the $-0.25 \mathrm{sec}$ group, but no significant differences from control for the $-0.5,-1.0$, or $-3.0 \mathrm{sec}$ ISI groups. Furthermore, no evidence of significant simultaneous ( $0.0 \mathrm{sec}$ ISI) or forward $(+0.25,+0.5,+1.0,+3.0 \mathrm{sec}$ ISI $)$ conditioning effects were obtained. Mean response levels during extinction are also presented in Figure 2. Dunnett's $t$ test indicated no significant differences between any of the experimental and control treatment results.

Trial effects are shown in Figures 3-5. These show that little change in responsivity to the $C S$ occurred in subjects receiving the control group treatment of unpaired CS-US presentations. In contrast, acquisition training in most paired groups resulted in some response level increases that for all but the $-0.25 \mathrm{sec}$ group were not statistically different from control group behavior. As expected from the mean extinction results described above, CS-alone (extinction) trials following acquisition appeared to produce control group-like behavior in the paired groups.

To make observations parallel to those conducted in the previous study (Durkovic and Damianopoulos, 1986), an analysis of variance was carried out on changes over trials, using the results of the $-0.25 \mathrm{sec}$ backward, the $+1.0 \mathrm{sec}$ forward, and the unpaired control treatment groups. Analysis of the data from the 6 test trials during acquisition indicated significant treatment 
Figure 2. ISI function curves for conditioning and extinction paradigms. Each point is the group mean TA reflex change from preacquisition response to $\mathrm{CS}$ alone trials $( \pm \mathrm{SEM})$. Conditioning, mean change of $6 \mathrm{CS}$-alone "probe" trials during acquisition. Extinction, mean change of $30 \mathrm{CS}$-alone trials following acquisition. Asterisk denotes significant differences from $+30 \mathrm{sec}$ control group response changes.

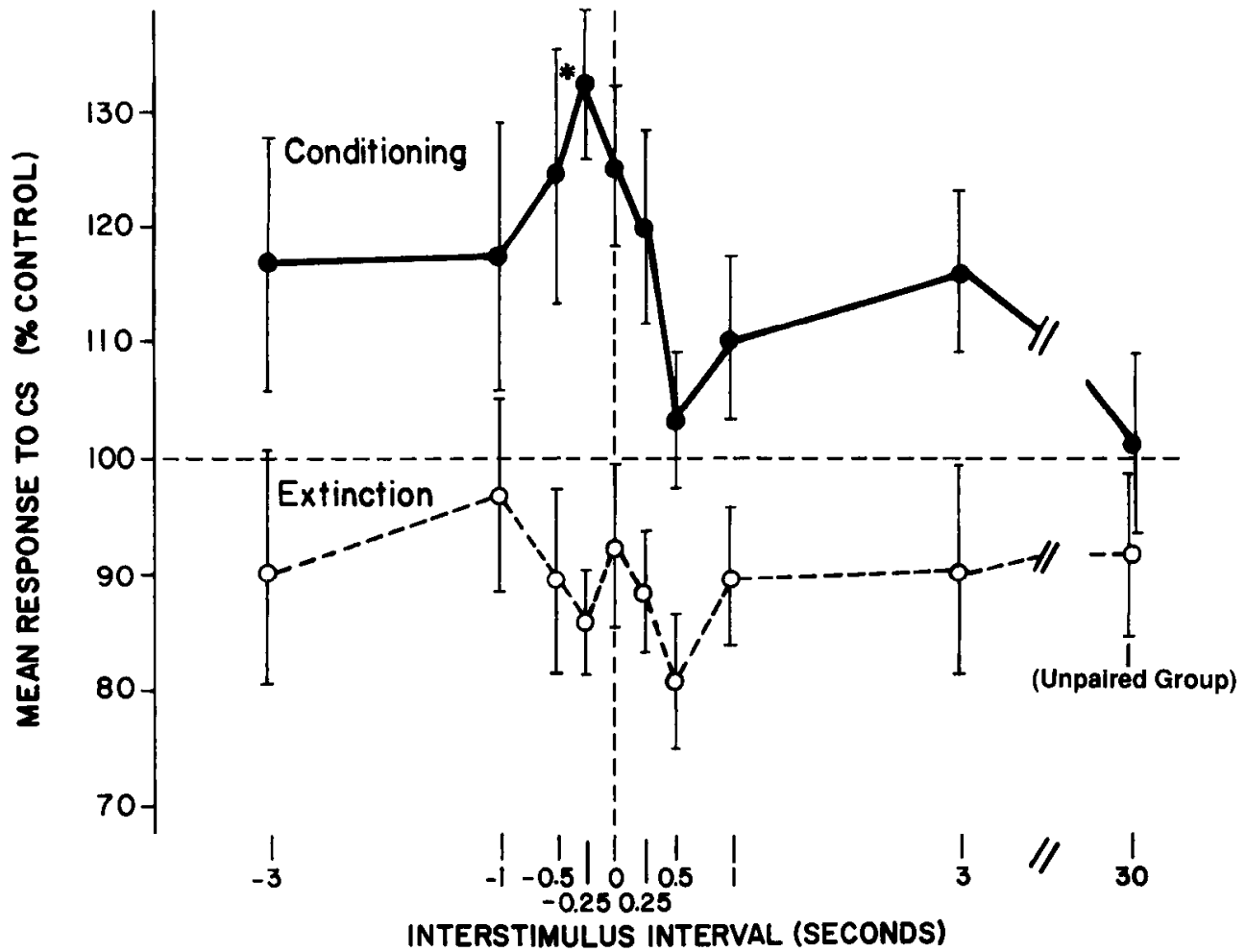

$(F(2,21)=3.47, p<0.025)$ and trial $(F(5,105)=4.20, p<$ $0.005)$, but no significant treatment $\times$ trial interaction $(F(10,105)=0.320, p>0.05)$ effects. Additionally, the overall mean differences between the $-0.25 \mathrm{sec}$ group and both the $+1.0 \mathrm{sec}$ group and the control group exceeded the critical difference (C.D. $=21.54$ at $p<0.05$ ). These data indicate that the $-0.25 \mathrm{sec}$ group response level was greater than those of both the $+1.0 \mathrm{sec}$ group and the control group over acquisition.

A similar analysis of the 5-trial block extinction data indicated no significant treatment effect $(F(2,21)=0.27, p>0.05)$, but the trial block $(F(5,105)=20.31, p<0.005)$ and treatment $\times$ trial block interaction $(F(10,105)=2.78, p<0.005)$ were significant. Since the extinction curves for the $+1.0 \mathrm{sec}$ group and the control group are very similar, the treatment $\times$ trial block interaction indicates that the slope of the $-0.25 \mathrm{sec}$ group over extinction is greater than the slope of the $+1.0 \mathrm{sec}$ and of the control groups, suggesting a more pronounced extinction effect for the BC group.

\section{Discussion}

The present results contribute evidence for the supposition that fundamental differences exist between the neural processes responsible for forward and backward conditioning in the spinal preparation. $\mathrm{BC}$ was demonstrated here to be elicited through spinal reflex pathways activated only by the large myelinated afferent fibers of the CS, while the same CS was unsuccessful in producing significant FC. In addition, the results are similar to previous observations that suggested that $\mathrm{BC}$ appears to be uncommonly influenced by extinction procedures compared to $\mathrm{FC}$ groups (Durkovic and Damianopoulos, 1986). This discussion centers around these findings and their implications for current theories of associative learning and for spinal conditioning as a simplified neural model of mammalian learning.

The results are best approached in the context of a comparison with a parallel study on this preparation in which a higherintensity CS (activation of large and small myelinated cutaneous afferent fibers) was employed (Durkovic and Damianopoulos, 1986). To help with this comparison, we constructed "net conditioning" (Fig. 6) and "net extinction" (Fig. 7) curves from the data of the 2 studies. Each point on these curves is the arithmetic difference between experimental and control group means for each paradigm for each study, and represents an attempt to reveal the pure associative effects for each ISI group by removing effects due to nonassociative factors (e.g., Light and Durkovic, 1977).

The backward and simultaneous portions of the net conditioning curves in Figure 6 show parallel behavioral effects for the 2 CS intensities, with declines on either side of the optimum ISI $(-0.25 \mathrm{sec})$. The similarities in these portions of the studies are maintained for the net extinction data as well (Fig. 7). The similarity of these ISI function curves suggests that most, or possibly even all, of the BC effects observed in the Durkovic and Damianopoulos (1986) study were brought about by spinal reflex pathways activated by A-alpha cutaneous fibers of the saphenous nerve. Any differences between the $\mathrm{BC}$ results of the 2 studies (e.g., BC acquisition effects were statistically significant in the -0.5 and $-1.0 \mathrm{sec}$ ISI groups in the earlier study) might be explained by (1) the fact that not all A-alpha cutaneous fibers of the saphenous nerve were activated by the CS in the present study (see Materials and Methods), in contrast to the earlier work, and/or (2) a contribution from A-delta fiber inputs to the $\mathrm{BC}$ results of the earlier work. In any case, the present results show that $\mathrm{BC}$ can take place over flexion reflex pathways activated only by A-alpha fibers of the saphenous nerve.

Unlike the backward and simultaneous portions of the ISI function curves, the $2 \mathrm{CS}$ intensities produce widely different results for FC, as is shown by the data in Figure 6 . While the present study indicated that no significant conditioning was ob- 


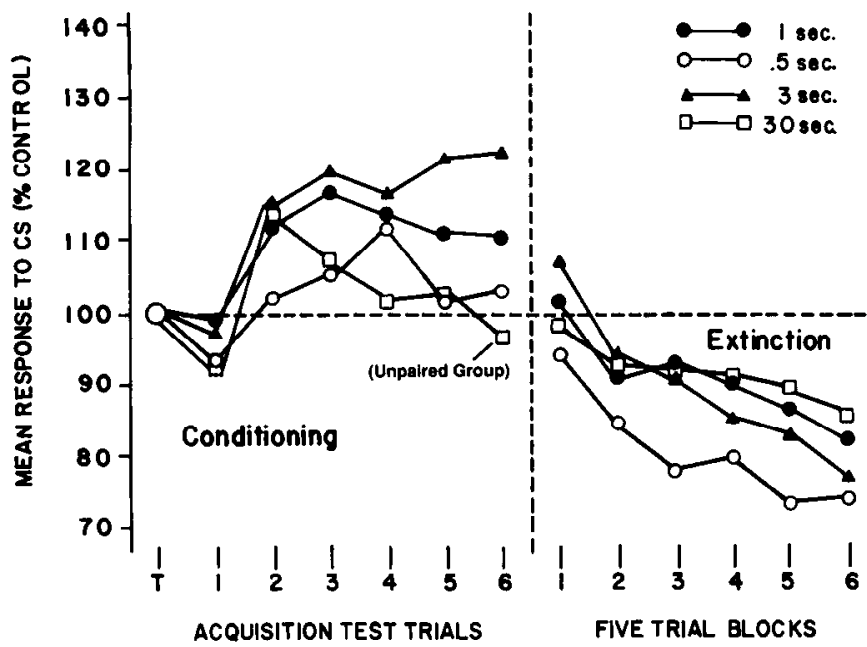

Figure 3. Trial effects during conditioning and extinction for the +3 , +1 , and $+0.5 \mathrm{sec}$ forward conditioning groups, and the $+30 \mathrm{sec}$ unpaired control group. $T$, Mean control response to preacquisition CSalone responses.

tained in any of the FC groups, the +0.5 and +1.0 sec groups of the earlier study exhibited clear conditioned reflex facilitation. While there is a suggestion of a contribution to forward pairing effects from the A-alpha circuitry, as indicated from inspection of the curves for FC groups in Figures 3 and 4, such effects are unreliable, as indicated by both the overall statistical tests (i.e., Dunnett's) and the ANOVA presented in Results. These results are in sharp contrast to similar tests of significance conducted on the FC data of the Durkovic and Damianopoulos (1986) study, in which the CS also recruited A-delta fibers.

That the spinal circuitry activated only by A-alpha cutaneous fibers of the CS does not contribute significantly to forward pairing effects is also supported by previous experiments on this preparation. For example, the CS intensity study by Misulis and Durkovic (1984) indicated that only unpaired control grouplike responses were induced in animals given paired trials when the CS activated only A-alpha cutaneous fibers. Even more im-

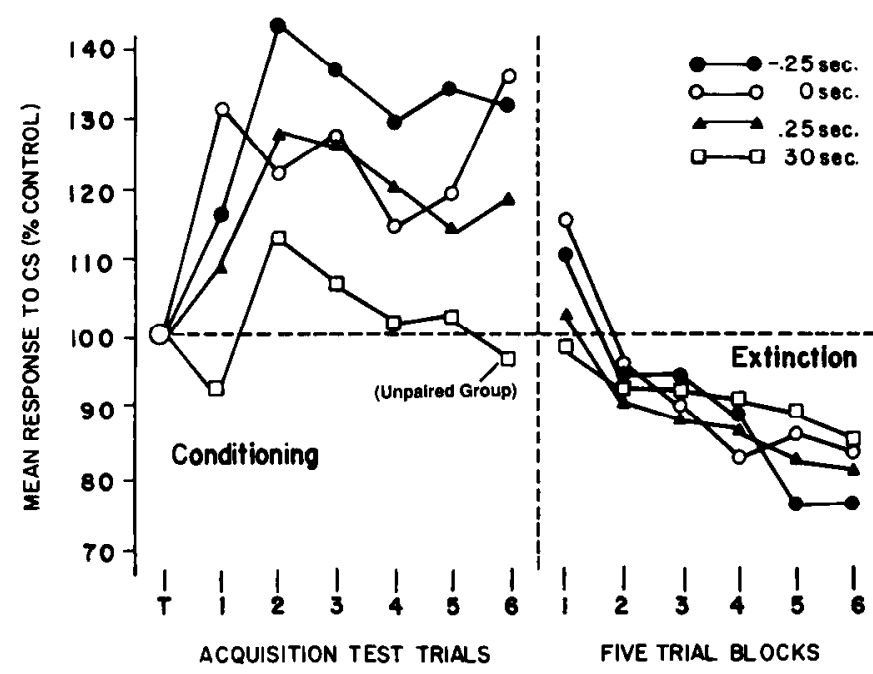

Figure 4. Trial effects during conditioning and extinction for the +0.25 , 0 , and $-0.25 \mathrm{sec}$ conditioning groups and the $+30 \mathrm{sec}$ unpaired control group.

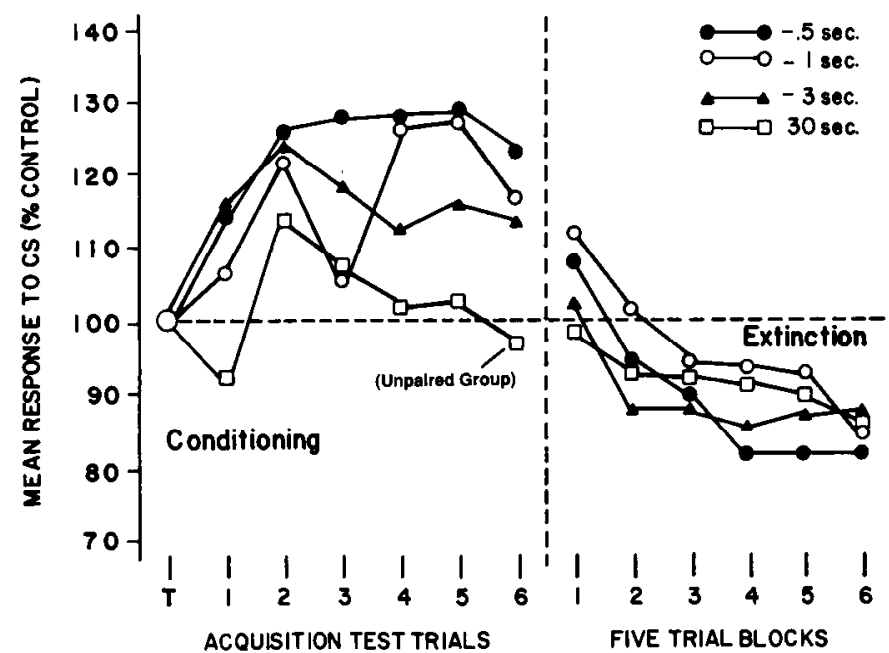

Figure 5. Trial effects during conditioning and extinction for the -0.5 , -1.0 , and $-3.0 \mathrm{sec}$ backward conditioning groups and the $+30 \mathrm{sec}$ unpaired control group.

portant, animals exhibiting pronounced conditioned facilitation induced with CSs that activated A-alpha and A-delta cutaneous fibers showed no facilitation effects when only the spinal circuitry activated by A-alpha cutaneous fibers of the CS was tested (Durkovic, 1983, 1987). These results strongly suggest that the FC observed in this preparation is elicited over spinal reflex pathways activated by A-delta cutaneous fibers of the saphenous nerve. Furthermore, the spinal reflex pathways activated only by $\mathrm{A}$-alpha saphenous nerve fibers appear to exert little influence on the development of FC. Thus, BC and FC may be brought about by the activation of different neural circuitries within the spinal cord. This possibility draws support from anatomical and physiological data that provide substantial evidence that A-alpha and A-delta fiber inputs to the spinal cord are, to a great extent, segregated-that is, they terminate in different spinal cord laminae (e.g., Brown, 1981; Price, 1986).

Alternative explanations for the forward pairing effects cannot

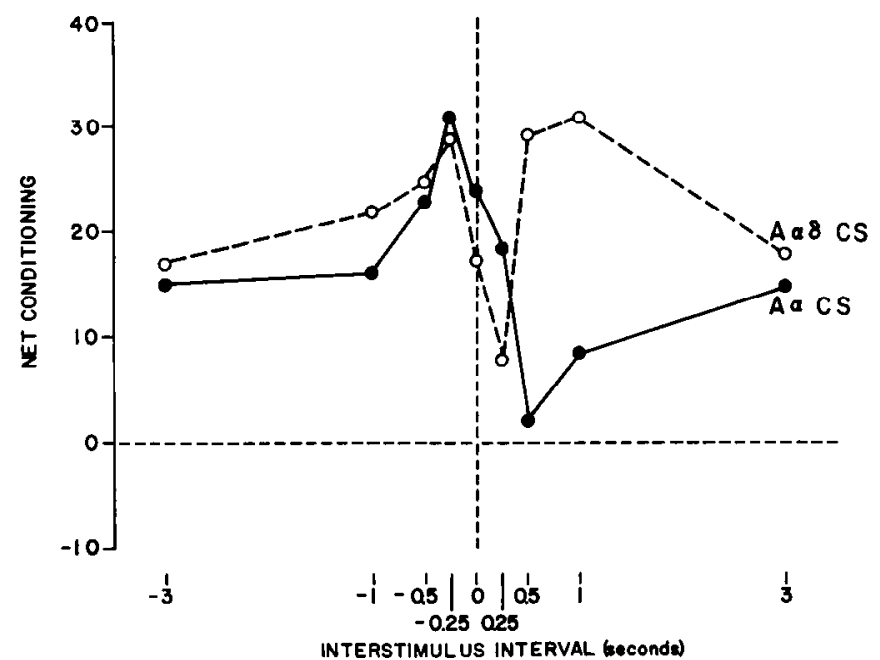

Figure 6. Net conditioning ISI function curves for $2 \mathrm{CS}$ intensities (see text). Net conditioning is the numerical difference between experimental and control group mean acquisition results (Light and Durkovic, 1977). Means were calculated from the results of the $6 \mathrm{CS}$-alone probe trials presented during acquisition. 


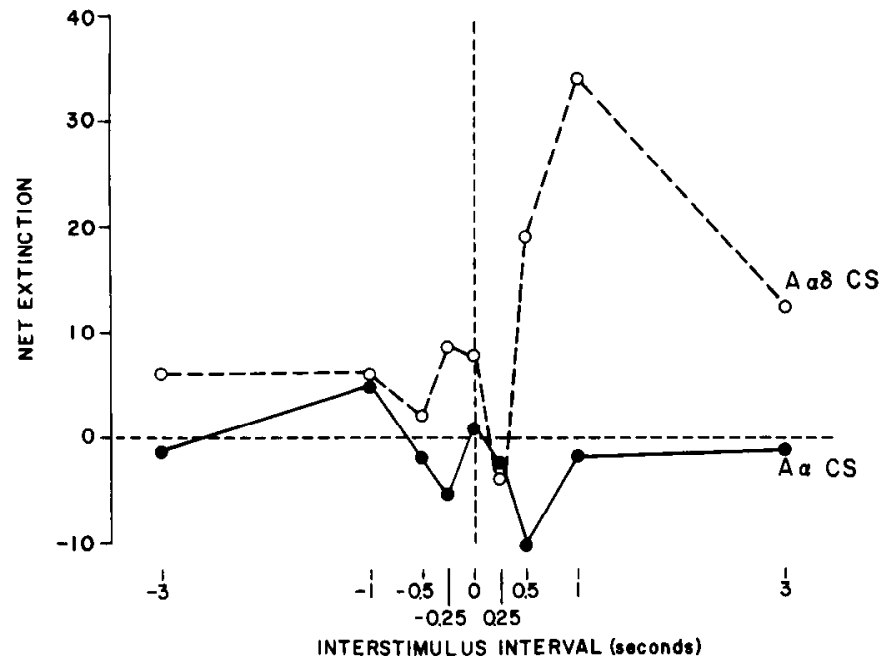

Figure 7. Net extinction ISI function curves for 2 CS intensities (see text). Net extinction is the numerical difference between experimental and control group 30-trial mean extinction results.

be excluded. For example, a group of "flexion reflex" interneurons could receive influences over pathways activated by both A-alpha and A-delta cutaneous inputs, and their activation for the production of the $\mathrm{FC}$ effect could require both inputs. In this case, the observed differences in CS intensity requirements for forward and backward conditioning might be viewed as a difference in threshold, rather than a true separation of 2 kinds of learning taking place over 2 kinds of neural circuits. However, a number of observations make this type of explanation unlikely. For example, a study on FC in this preparation showed that with an A-alpha-delta CS, conditioned reflex facilitation was obtained when an A-alpha-delta US was employed, but not when the US activated only A-alpha cutaneous fibers (Light and Durkovic, 1977). Because the superficial peroneal nerve (activation of which provides the US input) is larger than the saphenous nerve and was stimulated at a higher frequency, the A-alpha cutaneous inputs have a much greater impact on the spinal reflex circuits than do saphenous nerve (CS) inputs (as judged by reflex magnitude differences; see, e.g., Light and Durkovic, 1977) and might be expected to overcome such threshold requirements proposed for the CS inputs. Nevertheless, A-delta fiber activation was a requirement here too. Furthermore, evidence suggests that (1) single A-delta fiber activation elicits pain sensations in humans, while A-alpha fiber activation does not (Torebjörk and Ochoa, 1980); (2) A-alpha and A-delta fibers exhibit a specificity in synaptic connections based on the different carbohydrates on their cell surfaces (Mori, 1986); and (3) A-alpha and A-delta fibers release different transmitters/neuromodulators (Hunt, 1983; LaMotte, 1986). Thus, there appear to be features that are unique to the A-delta cutaneous inputs to the spinal cord. Altogether, these observations render a simple threshold explanation for the differences between CS requirements for $\mathrm{FC}$ and $\mathrm{BC}$ less compelling, and suggest that spinal circuitry differences may exist for $\mathrm{FC}$ and $\mathrm{BC}$.

Why $\mathrm{BC}$ involves $\mathrm{A}$-alpha cutaneous fiber activation by the $\mathrm{CS}$, and why activation of A-delta fibers appears to be influential primarily for FC, is unknown. However, the widespread and relatively long-lasting presynaptic inhibitory effects of cutaneous nerve activity (e.g., Eccles et al., 1962) make this a possible (and testable; e.g., Sastry, 1979) candidate as a neural mechanism involved in these observations and in influencing the form of the ISI function curves. A brief ( $<500 \mathrm{msec}$ ) period of presynaptic inhibition brought about by the initial part of the saphenous nerve A-delta fiber stimulus train, resulting in inhibition of A-delta superficial peroneal nerve output, has previously been proposed as a possible mechanism that prevents associative activation of reflex pathways required for conditioned reflex facilitation in certain conditioning groups (e.g., those with 0.0 and $+0.25 \mathrm{sec}$ ISIs; Durkovic, 1986). With somewhat different characteristics (e.g., slower onset, longer duration) it is not difficult to imagine how presynaptic inhibitory effects (from the saphenous nerve A-alpha activity) on A-alpha fibers of the superficial peroneal nerve might allow the observed $\mathrm{BC}$ effects, but prevent their associative influences in FC paradigms.

As can be seen in Figure 6, appropriately selected CS intensitics and optimal ISIs result in similar BC and FC net conditioning magnitudes. However, in examining the net extinction data in Figure 7, one can see that there are major differences between $\mathrm{BC}$ and $\mathrm{FC}$ in their resistance to extinction. The $\mathrm{BC}$ groups show little retention of conditioned reflex facilitation regardless of CS intensity, while some of the A-alpha-delta CS intensity FC groups resisted extinction (e.g., compare Dunnett's and ANOVA results for the 2 studies). In this regard, the apparently greater rate of decrease in response to CS-alone trials following acquisition for the $-0.25 \mathrm{sec}$ group of the present study (ANOVA) tends to support previous findings indicating behavioral differences between $\mathrm{BC}$ and $\mathrm{FC}$ effects during extinction (Durkovic and Damianopoulos, 1986). These behavioral differences, and the neural circuitry differences noted above, provide support for the hypothesis that different neural mechanisms may be involved in the production of forward and backward conditioning effects.

Such a hypothesis has important implications for theories of associative learning, particularly those that require that conditioning occur only when CS onset precedes US onset. However, if backward pairing produces associative effects through mechanisms different from those that produce forward pairing effects, such theories may not necessarily be invalidated by the existence of BC. Furthermore, consideration of $\mathrm{BC}$ as a process (or processes) different from $\mathrm{FC}$ might be an important step in rationalizing the apparent paradox of the existence of both excitatory (e.g., Heth and Rescorla, 1973; Spetch et al., 1981; Durkovic and Damianopoulos, 1986) and inhibitory (e.g., Plotkin and Oakley, 1975; Hall, 1984) BC effects, and in helping to interpret the meaning and significance of $\mathrm{BC}$.

With regard to the simplified preparation employed in these studies, the many major parallels between spinal conditioning characteristics and the conditioned responses of intact animals (e.g., Durkovic, 1975, 1983, 1985, 1986; Durkovic and Light, 1975; Patterson, 1975, 1980; Light and Durkovic, 1977; Misulis and Durkovic, 1982, 1984), including backward conditioning (Durkovic and Damianopoulos, 1986) and the technical advantages associated with the spinal preparation (e.g., Durkovic, 1986), suggest that spinal conditioning is a valid and useful modcl for rescarch on the ncural mcchanisms of mammalian learning and memory. Furthermore, research from other laboratories suggests that alterations at the spinal level participate in the production of certain learned motor responses in the intact animal (e.g., Wolpaw and Lee, 1987), so the results obtained from the spinal model may have general applicability.

Finally, the present discovery of backward conditioning effects produced within spinal reflex pathways activaled by 
A-alpha cutaneous fibers represents a potentially significant finding for neurophysiological studies of the spinal conditioning phenomenon. This is both because the recordings of A-alpha cutaneous fiber inputs at the cord level can more readily be correlated with cellular neurophysiological alterations in reflex output than can the slower-conducting A-delta fiber activity required for $\mathrm{FC}$, and because the neural model is simplified with the activation of only a single-size cutaneous fiber group by the CS.

\section{References}

Brown, A. G. (1981) Organization in the Spinal Cord, Springer-Verlag, New York, pp. 136-153.

Durkovic, R. G. (1975) Classical conditioning, sensitization and habituation in spinal cat. Physiol. Behav. 14: 297-304.

Durkovic, R. G. (1983) Classical conditioning of the flexion reflex in spinal cat: Features of the reflex circuitry. Neurosci. Lett. 39: 155160.

Durkovic, R. G. (1985) Retention of a classically conditioned reflex response in spinal cat. Behav. Neural Biol. 43: 12-20.

Durkovic, R. G. (1986) The spinal cord: A simplified system for the study of neural mechanisms of mammalian learning and memory. In Development and Plasticity of the Mammalian Spinal Cord, M. E. Goldberger, A. Gorio, and M. Murray, eds., Fidia Research Series, vol. III, pp. 183-192, Springer-Verlag, New York.

Durkovic, R. G. (1987) Associative LTP of selective spinal reflexes. Soc. Neurosci. Abstr. 13: 607.

Durkovic, R. G., and E. N. Damianopoulos (1986) Forward and backward classical conditioning of the flexion reflex in the spinal cat. J. Neurosci. 6: 2921-2925.

Durkovic, R. G., and A. R. Light (1975) Spinal conditioning: Unconditioned stimulus intensity requirement. Brain Res. 98: 364-368.

Durkovic, R. G., and S. Onifer (1985) Interstimulus interval effects on spinal conditioning using a low intensity CS. Soc. Neurosci. Abstr. 11: 1109.

Eccles, J. C., P. G. Kostyuk, and R. F. Schmidt (1962) Presynaplic inhibition of the central actions of flexor reflex afferents. J. Physiol. (Lond.) 161: 258-281.

Edwards, A. L. (1968) Experimental Design in Psychological Research, Holt, Rinehart and Winston, New York, p. 148.

Hall, J. F. (1984) Backward conditioning in Pavlovian type studies. Pavlov. J. Biol. Sci. 19: 163-168.

Heth, C. D., and R. A. Rescorla (1973) Simultaneous and backward fear conditioning in the rat. J. Comp. Physiol. Psychol. 82: 434-443.
Hunt, C. C., and A. K. McIntyre (1960) An analysis of fibre diameter and receptor characteristics of myelinated cutaneous afferent fibres in cat. J. Physiol. (Lond.) 153: 99-112.

Hunt, S. P. (1983) Cytochemistry of the spinal cord. In Chemical Neuroanatomy, P. C. Emson, ed., pp. 53-84, Raven, New York.

LaMotte, C. C. (1986) Organization of dorsal horn neurotransmitter systems. Spinal Afferent Processing, T. L. Yaksh, ed., Plenum, New York.

Light, A. R., and R. G. Durkovic (1977) US intensity and blood pressure effects on classical conditioning and sensitization in spinal cat. Physiol. Psychol. 5: 81-88.

Lundquist, E. F. (1953) Design and Analysis of Experiments in Psychology and Education, Houghton Mifflin, New York, pp. 266-273.

Misulis, K. E., and R. G. Durkovic (1982) Classically conditioned alterations in single motor unit activity in the spinal cat. Behav. Brain Res. 5: 311-317.

Misulis, K. E., and R. G. Durkovic (1984) Conditioned stimulus intensity: Role of cutaneous fiber size in classical conditioning of the flexion reflex in spinal cat. Exp. Neurol. 86: 81-92.

Mori, K. (1986) Lectin Ulex europaeus agglutinin 1 specifically labels a subset of primary afferent fibers which project selectively to the superficial dorsal horn of the spinal cord. Brain Res. 365: 404-408.

Patterson, M. M. (1975) Effects of forward and backward classical conditioning procedures on a spinal cat hind-limb flexor nerve response. Physiol. Psychol. 3: 86-91.

Patterson, M. M. (1980) Mechanism of classical conditioning of spinal reflexes. In Neural Mechanisms of Goal-Directed Behavior and Learning, R. F. Thompson, L. Hicks, and V. B. Shvrykov, eds., pp. 263272, Academic, New York.

Plotkin, H. C., and D. A. Oakley (1975) Backward conditioning in the rabbit (Oryctolagus cuniculus). J. Comp. Physiol. Psychol. 88: 586-590.

Price, D. D. (1986) The question of how the dorsal horn encodes sensory information. In Spinal Afferent Processing, T. L. Yaksh, ed., pp. 445-466, Plenum, New York.

Sastry, B. R. (1979) Presynaptic effects of morphine and methionineenkephaline in feline spinal cord. Neuropharmacology 18: 367-375.

Spetch, M. L., D. M. Wilkie, and J. P. J. Pinel (1981) Backward conditioning: A re-evaluation of the empirical evidence. Psychol. Bull. 89: 163-175.

Torebjörk, E., and J. L. Ochoa (1980) Specific sensations evoked by activity in single identified sensory units in man. Acta Physiol. Scand. 110: 445-447.

Wolpaw, J. R., and C. L. Lee (1987) Spinal localization of a primate memory substrate: Operantly conditioned $\mathrm{H}$-reflex asymmetry survives cord transection. Soc. Neurosci. Abstr. 13: 165. 